Jan Woleński

\title{
AGAINST TRUTH AS COHERENCE
}


I. Coherence theories of truth form a variety of approaches to the concept of truth which fall under the scheme

a proposition $\alpha$ is true if and only if

(a) $\alpha$ belongs to a system $\boldsymbol{S}$;

(b) $\boldsymbol{S}$ is a coherent system.

However, (1) can hardly be considered as a satisfactory definition, because it describes 'coherence theories' by 'coherent'. So (1) must meet the idem per idem (or ignotum per ignotum) objection. This was attempted by various particular coherence theories. ${ }^{1}$

Traces of the coherence theory (in the sense of (1)) are to be found in rationalistic epistemological systems of Leibniz, Spinoza, Kant, Hegel and Fichte (see [12]). However, the first fully advanced coherence theory was proposed by Bradley in XIX century. He was followed by Joachim, McTaggart, and particularly Blanshard and Ewing. The coherence theory later became popular among philosophers of the Vienna Circle, notably in Neurath and Hempel. ${ }^{2}$ Recently, Rescher tried to combine various proposals of coherentists into one fully unified view (see [7]). Let me add to this brief account of the development of the coherence theory that coherence was principally proposed as the very criterion of truth. So we can say that coherence theories define truth by its criterion. Usually, criterial definitions of truth are contrasted with the correspondence theory which describes truth without any reference to truth-criteria. ${ }^{3}$ The contrast between the correspondence and coherence theory is very often perceived as fundamental. According to Quine

Philosophy primers tell of two opposite doctrines as to the nature of truth: the coherence theory and the correspondence theory. ${ }^{4}$

\footnotetext{
1 The label 'coherence theories' (in singular and plural as well) occurs in this paper as an abbreviation for 'coherence theories of truth'. This reservation is important, because the word 'coherence' is recently used in the context 'coherence theories of knowledge'. Let me note in advance that my criticism of coherence theories goes only against truth as coherence.

${ }^{2}$ However, let me note that not all philosophers of the Vienna Circle accepted this theory. Schlick is perhaps the most notable example. Carnap's view evolved from the coherence theory to the semantic one.

3 The evidence theory and the pragmatic theory are another examples of criterial truth-definitions.

4 [6], p. 214. It should be noticed that on Quine's view these two theories are not gen-
} 
This suffices, I think, to show that the coherence theory should be seriously taken.

There are various possibilities of how coherence theories could be classified. Walker (in [12]) proposes to divide them into pure and mixed. A coherence theory is pure if it proposes coherence as the only criterion of truth, but a coherence theory is mixed if it combines coherence with something else. Usually, mixed coherence theories proceed by distinguishing a set, say $\boldsymbol{P}$, of privileged statements and then defining a coherent system as an extension of $\boldsymbol{P}$. Bradley's theory of truth is an example of the pure coherence theory. On the other hand, coherence theories which cabined coherence with acceptance of some basic statements by experts. ${ }^{5}$

I suggest we distinguish normal from Bradleyian coherence theories. A coherence theory is said to be normal if and only if it is based on a logic (classical or not) which is analyzable by standard metalogical means. ${ }^{6}$ A coherence theory is Bradleyian if and only if uses a special logic accepted by Bradley himself and other neo-Hegelians. The theories of Bradley and his followers are Bradleyian; the rest are normal. ${ }^{7}$ I will argue that coherence theories are either (a) too obscure from the metalogical point of view, or (b) they do not realize their fundamental promises, or still (c) they are redundant. The case (a) is directed against Bradleyian theories, whereas (b) and (c) against normal ones. If I am right, the situation of coherence theories is not good. Following a reasoning of Calif Omar on the occasion of ordering that the Alexandrian Library should be burnt ${ }^{8}$, we can restate the situation by

uine rivals, because the coherence theory concerns truth-criteria, but the correspondence theory says how truth is related to what true propositions are about.

${ }^{5}$ See [4]. Of course, we have several other possibilities, e.g. to combine coherence with evidence.

${ }^{6}$ Of course, this criterion is not quite precise. By standardness, I understood here the applicability of such notions as consistency, compactness, completeness, independence, etc. in their usual meaning. This will be illustrated by examples in the course of this paper. Moreover, let me note that my argument are based on the assumption that classical logic is a formal skeleton for the concept of truth. This does not mean that it must be. However, I do not enter into the consequences of looking at the logic of truth by non-classical logic. However, see note 17.

7 At first sight, Rescher's case seems to invites us to introduce the third rubrics: a compromise between normal and Bradleyian theories. However, I think that Rescher's coherence theory fairly belongs to normal theories. It is, so to speak, an attempt to "civilize" (or to "barbare", if you want) Bradley's ideas by normal logic. See also note 21.

8 Actually, Omar's argument is rather a legend than a real historical fact. Neverthlesness, it is very impressive. 
if a theory is obscure, it should be abandoned; if it does not satisfy its promises, it should also be abandoned, and the same holds for a redundant theory. Since the coherence theory is obscure or it does not satisfy own promises or it is redundant, it should be abandoned.

II. According to Bradley, the whole knowledge-system is the bearer of truth. This is an epistemological counterpart of Bradley's holistic ontology which regards the whole reality as existing in the proper sense. Particular propositions may be at most partially true; this is his famous doctrine on the degrees of $\operatorname{truth}^{9}$. This idea raises the first serious problem. If the Bradleyian proposal is to be completely realized, it should supplemented by an explicit "logic of truth-degrees". We should know how to calculate the degree of truth for $\alpha \vee \beta$ or $\alpha \wedge \beta$ (or any other compound proposition), provided that degrees for $\alpha$ and $\beta$ are given. Speaking more generally, on the standard logic, we have

$$
X \in \mathbf{V E R} \Longleftrightarrow \forall_{\alpha \in X} v(\alpha)=1
$$

This means: $X$ is a true set if and only if $X$ exclusively consists of true propositions (each member of $X$ has truth as its logical value). ${ }^{10}$ Now let $\boldsymbol{B}, \mathbf{V E R}_{\mathrm{B}}$ and $1_{\mathrm{B}}$ denote the Bradleyian knowledge-system (the set of all Bradleyian truths), the Bradleyian truth-property and partial truth in the Bradleyian sense respectively. Obviously, the membership sign is replaced by equality for $\mathbf{V E R}_{\mathrm{B}}$ is unique,

$$
\boldsymbol{B} \in \mathbf{V E R}_{\mathrm{B}} \Longleftrightarrow \forall_{\alpha \in \boldsymbol{B}} v(\alpha)=1_{\mathrm{B}}
$$

does not hold under the Bradleyian principles, because particular propositions are only partially true. However, perhaps one would want to know how what amount of truth is sufficient for regarding a particular $\alpha$ as a member of $\boldsymbol{B}$. As far as I know, nobody of the Bradleyians has offered even a rough sketch of such a logic. The Bradleyians could hardly appeal to many-valued or probabilistic logic to solve this problem, because no satisfactory intuitive

\footnotetext{
9 Some Bradleyians (particularly, McTaggart) see no reasons to reject the correspondence theory. They say that Truth and Reality (both in the Bradleyian sense) are in mutual correspondence. However, this sense of the concept of correspondence is peculiar and it does not fit traditional intuitions connected with it. So I will disregard this interpretation of Bradley's theory as a sort of correspondence theory.

10 The symbols VER and 1 are distinguished because they refer to various entities: VER to set of propositions, but 1 to particular propositions.
} 
interpretation of these logics has been proposed so far. Since hopes for the future cannot be excluded, I do not stress this point as the main one.

However, there are more basic unclarities in the Bradleyian logic which allow several complaints against it. For instance, Russell says:

The coherence-theory is generally advocated $[\ldots]$ in the connection with logic entirely different from ours. ([10], p. 149)

Another author declares:

The first and principal difficulty of the coherence theory is to understand it. ([13], p. 155)

Woozley's statement is certainly exaggerated, because most philosophers do understand the Bradleyians, at least to some extent. I would like to continue Russell's pattern and focus on peculiarities of the Bradleyian logic, or rather the Bradleyian metalogic. Of course, I do not suggest that my objections against the Bradleyian logic are exactly the same which Russell has in his mind.

Perhaps the most extensive explanation of logic of the Bradleyian theory of truth is due to Blanshard (see [2]). He defines the coherent system as (a) consistent, (b) comprehensive, and (c) internally interconnected. We can assume that consistency is taken in its standard meaning in (a). ${ }^{11}$ Also the concept of comprehensiveness is relatively simple. It means that the coherent system covers the entire realm of truth. The most mysterious point of (a)-(c) is the last. Let me quote two explanations of what interconnectedness looks like:

It is perhaps in such systems as Euclidean geometry that we get the most perfect examples of coherence that have been constructed. If any proposition were lacking, it could be supplied from the rest; if any were altered, the repercussions would be felt through the length and breadth of the system. Yet even such a system as this falls short of ideal system. Its postulates are unproved; they are independent each other, in the sense that none of them could be derived from any other or even from all the others together; its clear necessity is bought by an abstractness so extreme as to have left out nearly everything what belongs to the character of actual things. A completely satisfactory system would have none of these defects. No proposition would be

11 I disregard here some doubts at this point which might appear when we take into account that the concept of consistency functions in Bradleyian theories in a specific "metalogical" context. It may be that this context modifies the standard sense of the concept of consistency. See also Appendix in the end of this paper. 
arbitrary, every proposition would be entailed by the others jointly and even singly, no proposition would stand outside the system. ([2], v. 2 , p. $265-266)$

$[\ldots]$ no set of propositions within the whole set is logically independent of all propositions in the remainder of the set [...] In the first place, in so far as they [propositions - J. W.] fulfil the coherence ideal, they are so related that any other proposition in the set follows with logical necessity if all the other propositions in the set are true. ([4], p. 229-230)

Both quotations show that it is unclear how some of Bradley's ideas should be understood. Blanshard and Ewing apparently use the standard metalogical vocabulary: they speak about axioms, entailment, independence, etc. However, Blanshard radically changes meaning of the term 'axiom', because, on the standard meaning, axioms of a system, say $\boldsymbol{S}$, are just those propositions, which are assumed not proved in $\boldsymbol{S} .{ }^{12}$ Moreover, independence is, at least on the standard metalogic, decisively a virtue, not a defect, of any system of axioms. Ewing's declaration is certainly clearer than that of Blanshard, but Ewing's case also requires further explanation. Is he talking about syntactic or semantic consequence? Obviously, his claim is correct (modulo the standard metalogic) only when his entailment is understood as semantic consequence; the same remark might be addressed to Blanshard's statement on "entailing [...] jointly and even singly".

Apart from those general points, there is something more particular to be raised in the connection with the Bradleyian logic.

Assume that the first-order classical logic is the logic of "our" truth. Then we have

$$
\begin{aligned}
& \forall_{X \subseteq \text { Prop }}\left[\forall_{Y \subseteq X}(Y \in \text { Fin } \Rightarrow Y \in \text { VER }) \Longrightarrow X \in \mathbf{V E R}\right] \\
& \forall_{X \subseteq \text { Prop }}\left[X \in \text { VER } \Longrightarrow \forall_{Y \subseteq X} \quad Y \in \text { VER }\right] .
\end{aligned}
$$

In words: (5a) if $X$ is a set of propositions and every finite subset of $X$ is true, then $X$ is also true; (5b) if $X$ is true, then every subset of $\mathrm{X}$ is also true. (5a) says that truth-property is compact. The reverse property which is expresssed by (5b) may be called 'down-compactness'. (5a) has no sense under Bradleyian metalogic, because no finite set of propositions belongs to $\mathbf{V E R}_{\mathrm{B}}$

$$
\forall_{X \subseteq \text { Prop }}\left(X \in \mathbf{F i n} \Rightarrow X \notin \mathbf{V E R}_{\mathrm{B}}\right) .
$$

\footnotetext{
12 Formally speaking, axioms of a formal system are, under the standard definition of proof, provable from the empty set (plus laws of logic). This definitions allows us to regard axioms as provable formulas.
} 
Thus, compactness holds trivially for $\mathbf{V E R}_{\mathrm{B}}$, because the antecedent of (5a) is always false. This means that properties of subsets of $\mathbf{V E R}_{\mathrm{B}}$ have no influence on the entire set. Moreover

$$
\left.\forall_{X \subseteq \text { Prop }}\left[X \in \mathbf{V E R}_{\mathrm{B}} \Longrightarrow \neg \exists_{Y \varsubsetneqq X} Y \in \mathbf{V E R}_{\mathrm{B}}\right)\right] .
$$

The reason for $(6 \mathrm{~b})$ is this. Assume that $\mathrm{X}$ is true in the Bradleyian sense. Take any subset of $\mathrm{X}$ which is different (to avoid a trivial case) from $X$ itself. Clearly, $Y$ does not belong to $\mathbf{V E R}_{\mathrm{B}}$, because only the full $\mathrm{X}$ is Bradleyian-true. The lack of not-trivial compactness is no peculiarity here, because many other sets of truths, the set of second-order truths, for instance, are even non-compact. However, the lack of downward-compactness is surely a peculiarity of Bradleyian truth. Since down-compactness seems to be a very desired property of truth, this peculiarity constitutes in my opinion a very serious defect of Bradleyian metalogic. ${ }^{13}$

The Bradleyians can respond to this criticism by pointing out that imposing our principles on the Bradleyian logic is not fair and deforms its essentials features. However, I do not think that this answer is satisfactory. Metalogical principles are too serious deal for any logic to leave them to very vague intuitions. At first, first, the Bradleyians should make clear what they propose instead of down-compactness. Their metalogic could then be taken seriously. As long as the matter is left as it now stands, we are fully entitled to condemn the Bradleyian coherence theory as obscure.

I now turn to normal coherence theories. According to Neurath:

In unified science we try to construct a non-contradictory system of protocol saentncs and non-protocol sentences (including laws) [...] When a new protocol sentence is presented to us we compare it with the system at our disposal, and determine whether or not it conflicts with that system. If the sentence does conflicts with the system, we may discard it as useless (or false), as for instance, would be done with "In Africa lions sing only in major scales". One may, on the other hand, accept the sentence and so change the system that it remains consistent even after the adjunction of the new sentence. The sentence would then be called "true". ${ }^{14}$

\footnotetext{
13 See also Appendix on the end of this paper. What I call here down-compactness, contrary to compactness, is not an important metalogical property. But perhaps these considerations show an interesting philosophical application of it.

14 [5], p. 203 (page reference to Eng. tr.).
} 
There is a popular argument, raised by Russell and Schlick, against normal coherence theories which points out that there are many systems which are mutually inconsistent but internally consistent. ${ }^{15}$ Schlick's version is this:

If one is to take coherence seriously as a general criterion of truth, then one must consider arbitrary fairy stories to be as true as historical reports, or as statements in a textbook of chemistry, provided that the story is constructed in such a way that no contradiction ever arises. I can depict by help of phantasy a grotesque world full of bizarre adventures: the coherence philosopher must believe in the truth of my account provided only I take care of the mutual compatibility of statements. $^{16}$

The merit of this argument is very clear. Assume that $X$ and $Y$ are internally consistent but mutually inconsistent sets of propositions. This means that there is a proposition, say $\alpha$, such that it belongs to $X$ if and only if its negation belongs to $Y$. Now, according to the normal coherence theory, both $\alpha$ and $\neg \alpha$ are true, because both belong to consistent systems of propositions. However, this is impossible unless we decide to drop the principle of contradiction. ${ }^{17}$ This argument may be put in a more formal manner. Let $\mathrm{X}$ be a consistent set of propositions. We say: ${ }^{18}$

(a) $X$ is branchable at a formula $\alpha$ if and only if both sets $X \cup\{\alpha\}$ and $X \cup\{\neg \alpha\}$ are consistent;

(b) $X$ is branchable if and only if there is a formula at which $X$ is branchable.

Now the following result is important:

$$
X \text { is branchable if and only if } X \text { is incomplete. }
$$

We can assume that if $X$ expresses our knowledge, it contains arithmetic. Due to Gödel's first incompleteness theorem, $X$ is incomplete. Then, via (8), we obtain that $X$ is branchable. Applying (7a), we conclude that there are two mutually inconsistent but internally consistent sets of propositions. Now,

\footnotetext{
${ }^{15}$ See [9] and [11]. In fact, Russell and Schlick direct this argument against all coherence theories, not only normal ones. However, for metalogical reasons, I consider it only in the context of normal theories.

16 [11], p. 215-216 (page reference to Eng. tr.).

17 Recall that we assume classical logic. Perhaps paraconsistent logic provides another perspective at this point.

18 This terminology is borrowed from [1].
} 
if $\alpha$ is a formula at which $X$ is branchable, $\alpha$ is both true and false. If we carefully inspect the Russell-Schlick argument against the coherence theory, we very quickly find its important premise: coherence is entirely reducible to consistency. This premise is however inconsistent with the basic intentions of the coherentists, regardless whether they defend Bradleyian or normal theories. An important ingredient of any coherence theory is this: there are many internally consistent sets but one and only one of them is coherent. So we must look for another argument. Fortunately, we can concentrate on consistency.

Any normal coherence theory defines coherence as consistency plus "something" else; this "something" is assumed to be a selector of the unique coherent system from a variety of consistent sets of propositions. Schematically ( $\mathbf{C O H}$ - coherence, $\mathbf{C O N}$ - consistency),

$$
\mathrm{COH}=\mathrm{CON}+?
$$

where the question mark indicates "something else", for example a special relation to experience or acceptance by experts. (9) may be transformed into

$$
X \in \mathbf{C O H} \Longleftrightarrow X \in \mathbf{C O N} \wedge X \in \text { ?. }
$$

Now observe that consistency is by (10) a necessary condition for coherence. This fact is stated by

$$
X \in \mathbf{C O H} \Longrightarrow X \in \mathbf{C O N} \text {. }
$$

Assume that we want to prove that $X$ is coherent. Due to (11) and monotonicity of provability expressed by

$$
(\alpha \Rightarrow \beta) \Longrightarrow[\operatorname{PROV}(\alpha) \Rightarrow \operatorname{PROV}(\beta)],
$$

proof of coherence of $X$ entails that this set is consistent; to obtain this result, it is sufficient to put ' $X \in \mathbf{C O H}$ ' for $\alpha$ and ' $X \in \mathbf{C O N}$ ' for $\beta$. Now, the fact coherence saves a truth-criterion becomes an essential point. What should we require of truth-criteria? Certainly, their effectivity seems a quite reasonable requirement. (12) may be extended to effective provability:

$$
(\alpha \Rightarrow \beta) \Longrightarrow[\operatorname{EPROV}(\alpha) \Rightarrow \operatorname{EPROV}(\beta)]
$$

By putting once more ' $X \in \mathbf{C O H}$ ' for $\alpha$, ' $X \in \mathbf{C O N}^{\prime}$ for $\beta,(11)$ and detachment, we arrive

$$
\operatorname{EPROV}(X \in \mathbf{C O H}) \Longrightarrow \operatorname{EPROV}(X \in \mathbf{C O N}),
$$

which is equivalent to

$$
\neg \operatorname{EPROV}(X \in \mathbf{C O N}) \Longrightarrow \neg \operatorname{EPROV}(X \in \mathbf{C O H}) .
$$


Now, once more assume that $X$ contains arithmetic. By Gödel's second incompletess theorem, proof of consistency of $X$ is not effective. So proof of coherence of $X$ is not effective either. ${ }^{19}$ If we agree that replacing the correspondence theory by effective truth-criteria is one of promises of its rivals, we can conclude that coherence theories do not fulfil their own promises. ${ }^{20}$

The coherence theorists may drop the effectivity condition. ${ }^{21}$ and define consistency in a semantical way:

\section{$X$ is consistent if and only if it has a model.}

However, if so, the coherence theory weakened in this manner is identical with the semantic theory of truth. If so, the coherence theory is redundant. This concludes my criticism of the coherence theory as either obscure or going against own promises, or redundant. Quod erat demonstrandum. ${ }^{22}$

\section{Appendix}

Ilkka Niiniluoto raised an objection against my argument which points out that $\mathbf{V E R}_{\mathrm{B}}$ is not down-compact. The objection is this. One should not expect down-compactness of the Bradleyian truth, because it is comprehensive. So down-compactness of $\mathbf{V E R}_{\mathrm{B}}$ would result with failure of comprehensiveness of the Bradleyian truth. Yet the Bradleyian truth preserves consistency.

This is my reply. Let $\mathcal{M}$ be a model. A set $X$ of true propositions in $\mathcal{M}$ is comprehensive for $\mathcal{M}$ if and only if $X$ contains all propositions true in $\mathcal{M}$. It is easy to see that $X$ is comprehensive and down-compact as well. So $\mathbf{V E R}_{\mathrm{B}}$ behaves in a non-standard way. Now the problem is whether $\mathbf{V E R}_{\mathrm{B}}$ really preserves consistency (see note 11). Any further discussion is difficult unless the Bradleyians make clear their metalogic.

Acknowledgments. I wish to thank Elizabeth Brown of Boston University and Andrzej Pietruszczak of Torun University for their help in preparing this paper.

\footnotetext{
19 I assume that a metamathematical proof is effective, if it realizable, via aritmetization, in arithmetic. This is rather a moderate definition of effectivity.

${ }^{20}$ On several additional premises, this argument may be also used against the Bradleyian theories.

21 This seems to be in the case of Rescher's coherence theory; see [7] and [8]. The main reason for this evaluation is that Rescher (see [8]) defines coherence by coherence with a so-called ideal data-base which is taken in a semantic way.

${ }^{22}$ However, I think that Quine's view mentioned in note 4 pro vides a correct perspective of how the coherence theory is related to the correspondence one.
} 


\section{References}

[1] Asser, G., Einfuhrung in die mathematische Logik, Teil II, B. G. Teubner Verlagsgesselschaft, Leipzig 1972.

[2] Blanshard, B., The Nature of Truth, 2 vols., Allen and Unwin, London 1939.

[3] Ewing, A. C., Idealism: A Critical Survey, Methuen, London 1934.

[4] Hempel, C., "On the logical positivistic theory of truth", Analysis 2 (1934/5), 49-55.

[5] Neurath, O., "Protokollsätze", Erkenntnis 2 (1932/3), 204-214; Eng. tr. in A. J. Ayer, ed.: Logical Positivism, The Free Press, New York 1957, 199-208.

[6] Quine, W., Quiddities. An Intermittently Philosophical Dictionary, The Belknap Press, Cambridge, Mass. 1987.

[7] Rescher, N.: The Coherence Theory of Truth, Clarendon Press, Oxford 1973.

[8] Rescher, N., "Truth as ideal coherence", Review of Metaphysics, XXXVIII, 795-806.

[9] Russell, B.: Philosophical Essays, London, Longmans 1910.

[10] Russell, B., Theory of Knowledge The 1913 Manuscript, Allen and Unwin, London 1984.

[11] Schlick, M., "Über das Fundament der Erkenntnis", Erkenntnis 3 (1934), 79-99; Eng. tr. in A. J. Ayer, ed.: Logical Positivism, The Free Press, New York 1957, 209-227.

[12] R. Walker, The Coherence Theory of Truth, Realism, Anti-Realism, Routledge and Kegan Paul, London 1989.

[13] Woozley, A., The Theory of Knowledge, Hutchinson, London 1949.

JAN WOLEŃSKI

Institute of Philosophy

Jagiellonian University

Grodzka 52

31-044 Kraków, POLAND 